\title{
Open-eye detection using iris-sclera pattern analysis for driver drowsiness detection
}

\author{
ARUN D PANICKER and MADHU S NAIR* \\ Department of Computer Science, University of Kerala, Kariavattom, Thiruvananthapuram 695581, India \\ e-mail: arundp@gmail.com; madhu_s_nair2001@yahoo.com
}

MS received 18 June 2015; revised 4 February 2017; accepted 19 March 2017; published online 6 October 2017

\begin{abstract}
This paper proposes a novel approach for open-eye detection that can be used in driver drowsiness analysis based on computer vision techniques. The proposed method captures the driver video using a lowresolution camera. The proposed drowsiness detection system has three main stages. The first stage is face detection using elliptical approximation and template matching techniques. In the second stage, the open eye is detected using the proposed iris-sclera pattern analysis method. In the third stage, the drowsiness state of the driver is determined using PERcentage of eye CLOSure (PERCLOS) measure. The entire system is designed to be independent of any specific data sets for face or eye detection. The proposed method for open-eye detection uses basic image processing concepts of morphological and laplacian operations. The proposed system was evaluated with real-life images and videos. Open-eye detection accuracy of 93\% was achieved.
\end{abstract}

Keywords. Driver drowsiness; open-eye detection; iris-sclera pattern; morphological operations; PERCLOS.

\section{Introduction}

Driver drowsiness is a significant factor in a large number of vehicle accidents. Drowsy driving increases the risk of causing an accident four to six times, compared with alert driving [1-3]. In fact, at least $15-20 \%$ of all vehicle accidents have been estimated to be sleepiness related [4]. Drowsiness can result from sleep-related or task-related fatigues [5]. The development of technologies for detecting or preventing drowsiness at the wheel is a major challenge in the field of accident avoidance systems [6].

Diverse techniques have been developed for monitoring driver drowsiness. These techniques can be generally classified into three main categories [7]. The first category includes methods based on biomedical signals, like cerebral, muscular and cardiovascular activity [8-11]. Usually, these methods require electrodes attached to the driver's body, which will often cause annoyance to the driver. Also, long time driving would result in perspiration on the sensors, diminishing their ability to monitor accurately.

The second category includes methods based on the driver's performance, by monitoring the steering wheel movement, accelerator or brake patterns, vehicle speed, lateral acceleration, lateral displacement and other signals recorded by CAN [12-15]. The advantage of these approaches is that the signal is meaningful and the signal acquisition is quite easy.

*For correspondence
However, these systems are subject to several limitations such as vehicle type, driver experience, geometric characteristics and condition of the road. Since these procedures require a considerable amount of time to analyse user behaviours, they do not work with the so called microsleeps, i.e., when a drowsy driver falls asleep for a few seconds on a very straight road section without changing the vehicle signals.

In the third category, computer vision uses natural and non-intrusive techniques for monitoring driver's drowsiness from the images captured by cameras placed in front of the user. These approaches effectively measure physical changes of the driver such as sagging posture, leaning of the driver's head and the open/closed states of the eyes. Different kinds of cameras and analysis algorithms have been reported in the literature for this approach: methods based on visible spectrum camera $[16,17]$; methods based on infra-red (IR) camera [7, 18-20] and methods based on stereo camera.

Methods for drowsiness detection based on computer vision involve face detection, eye detection and eye state determination. Several methods [21] have been developed for face detection using near IR [22, 23], neural networks [24-26], skin colour [27, 28], Gabor filters [29] and template matching (GFT) [30-32]. Methods based on skin colour may be restricted to specific ethnic groups or may fail under varying illumination conditions. The Viola-Jones method (VJM) [33] uses haar-like features for face detection. Even though it enables faster detection, results depend on the data set used for training the features. 


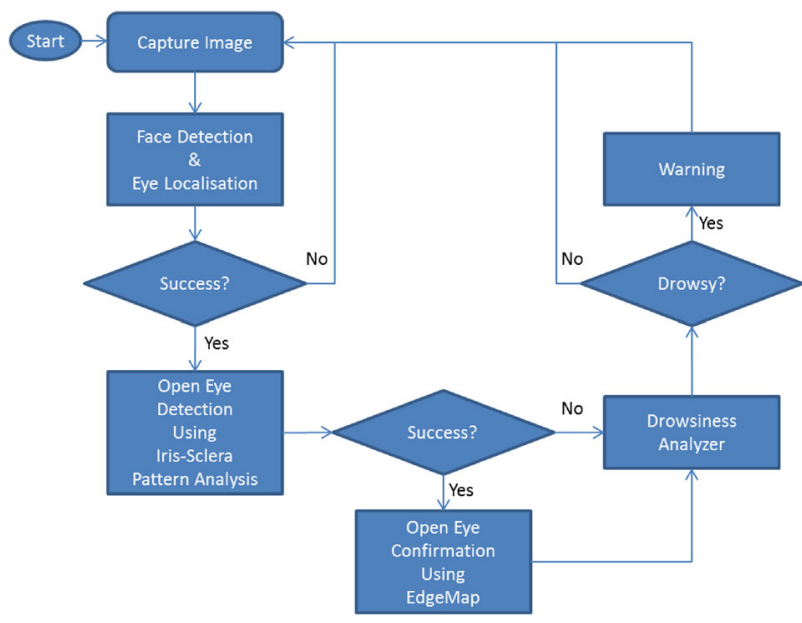

Figure 1. Proposed system architecture.

Various approaches exist in literature for eye detection and eye state determination using distance between eyelids [34], iris detection [35] and eyelid movement [36, 37]. Methods based on eyelid information do not deliver satisfactory results on dark skins and in low illuminations, and the iris methods require images of higher resolution for good results. Gabor filters could be used for eye detection [29, 38] and machine learning [29] for determining eye state. The downside of these methods is the computational overhead and the challenge in finding a reasonably good data set for training the open and closed eyes. Hence a new method is proposed for detecting open eyes in low-resolution images using morphological techniques.

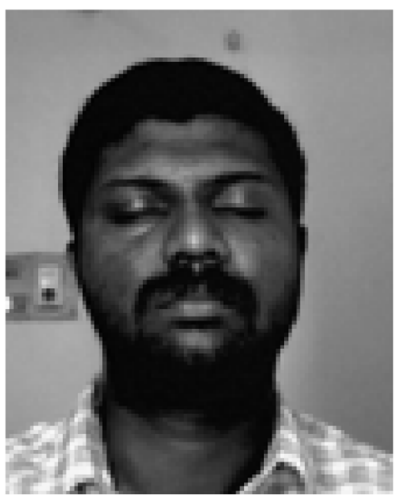

(a)

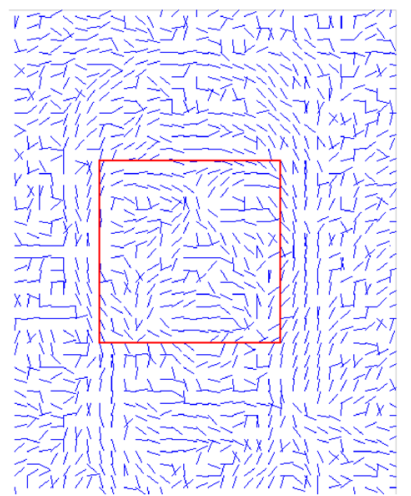

(b)
Figure 4. Fine face detection: (a) coarse face detected and (b) direction vector showing template-matched region for fine face.

In this paper, a new method is proposed for real-time driver drowsiness detection, based on computer vision techniques using a low-cost camera and a standard personal computer. A web camera is placed in front of the driver to monitor PERcentage of eye CLOSure (PERCLOS), the drowsiness parameter characterised by the maximum confidence [7], in real time and in a robust and automatic way. The proposed method does not need a calibration process and includes techniques to overcome the typical problems of image processing algorithms such as illumination change, user appearance and quick movements. For evaluation of the proposed system, several experiments have been designed with various illumination conditions and

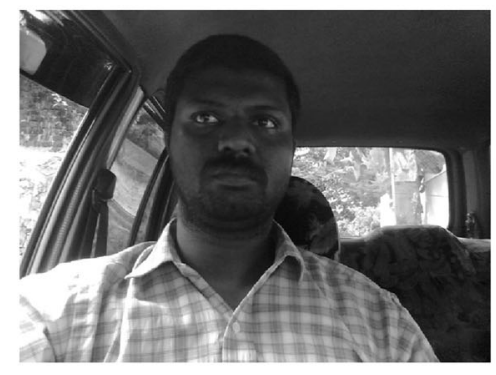

(a)

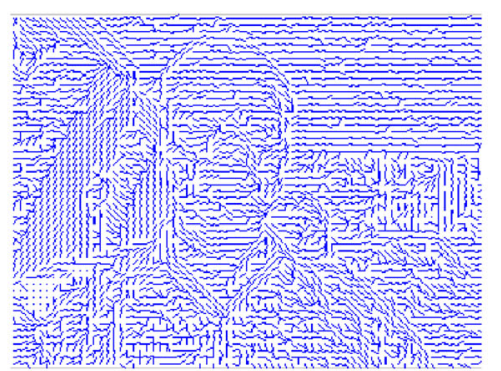

(b)

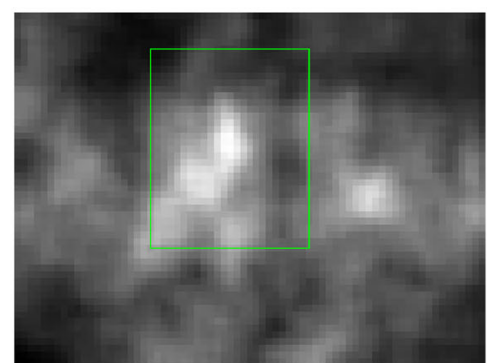

(c)

Figure 2. Steps in coarse face detection stage: (a) original image, (b) coarse directional image and (c) Hough accumulator.

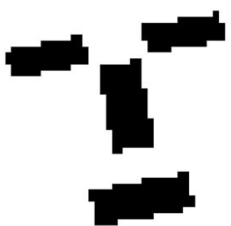

(a)

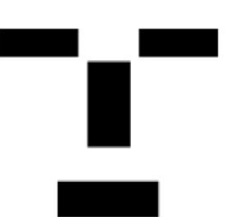

(b)

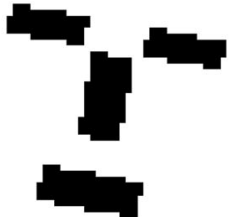

(c)

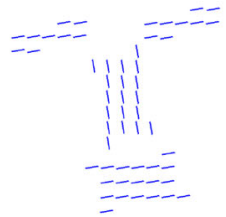

(d)

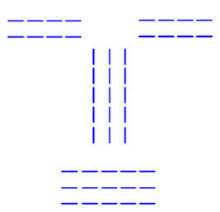

(e)

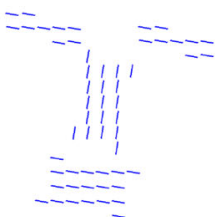

(f)

Figure 3. Template construction for different rotation angles. 
driver postures and appearances. Hence, the major contribution of this work is a novel method for detecting open eyes developed based on iris-sclera pattern analysis (ISPA).

In section 2, the proposed system architecture is defined. Section 3 describes drowsiness detection, dividing the system into face detection, proposed ISPA and PERCLOS. In section 4 the results and analysis of experimentation on real-life image and video data sets are presented. Finally, section 5 presents conclusions and discusses future research.

\section{Proposed system architecture}

The proposed method for drowsiness detection is split into three stages requiring only grey-level information, and solves the restrictions of other methods mentioned in section 1. The three stages are face detection, open-eye detection and PERCLOS estimation. The dataflow is shown in figure 1.

In the first stage, for face detection, the Perez-LazcanoEstévez (PLE) [35] approach is used with a modified set of templates, which is briefly explained in section 3.1 .

The proposed method of ISPA is used in the second stage for open-eye detection. In the proposed approach, the eye detection and eye state determination stages, performed as two processes in other implementations, are integrated into a single process by searching for the presence of an open eye. Thus eye states are identified with a minimum computation.

The drowsiness is determined in the third stage based on PERCLOS measure. The duration of closed eye is categorised as blink or PERCLOS based on the number of frames. If the PERCLOS state is more than $50 \%$ in the last $30 \mathrm{~s}$, a warning is triggered.

\section{ISPA method}

In the proposed method, open eyes are searched and the absence of an open eye indicates either that the eye is closed or inattention of the driver. A new method is proposed for detection of an open eye using the ISPA based on the symmetry of sclera around iris. The open-eye detection is applied on the localised eye region from the face image. The ISPA for open-eye detection also incorporates a part of PERCLOS method, which makes the drowsiness detection easier.

\subsection{Face detection and eye localisation}

The method proposed by Perez, Lazcano and Estévez [35] was used for face detection in this system with a modified set of templates. In this approach, face detection is achieved in two steps: coarse face detection and fine face detection. In both these steps, directional image is computed using the method proposed by Donahue and Roklin [39].

3.1a Coarse face detection: In this step, an approximate face location within the image is determined. Hough transform for an ellipse is used for face localisation based on the method proposed by Maio and Maltoni [32]. Figure 2 shows the coarse face detection steps for face localisation.

3.1b Fine face detection: The goal of this step is to detect the face location by determining the face rotation angle and size. This step is further divided into region of interest selection, face templates and fine detection.

Region of interest selection A higher resolution (compared with coarse face detection) directional image $I_{H R D}$ is computed using a smaller size local window (neighbourhood) [32]. The region of interest $I_{R O I}$ for the fine face

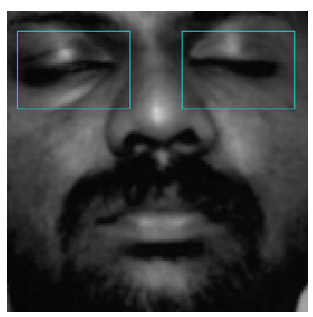

Figure 5. Selection of eye regions from the localised face region.

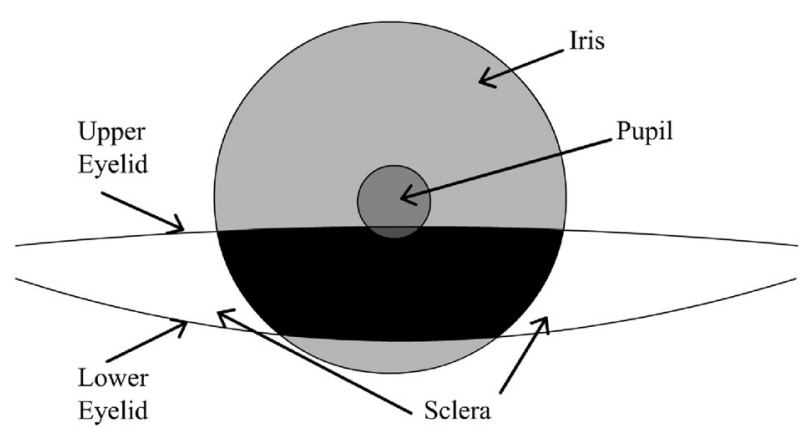

Figure 6. Computation of PERCLOS eye by determining the percentage of iris covered by eyelids and thus estimating visible iris region. (a)

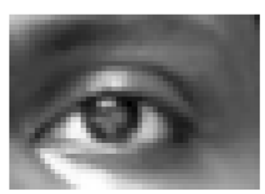

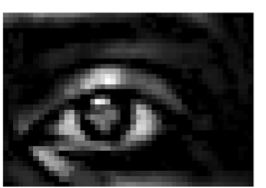

(b)

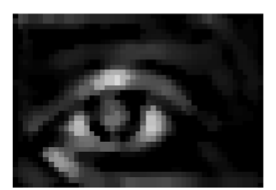

(c)
Figure 7. Preprocessing of the localised eye region: (a) eye region, (b) output of tophat transform and (c) after applying the median filter. 


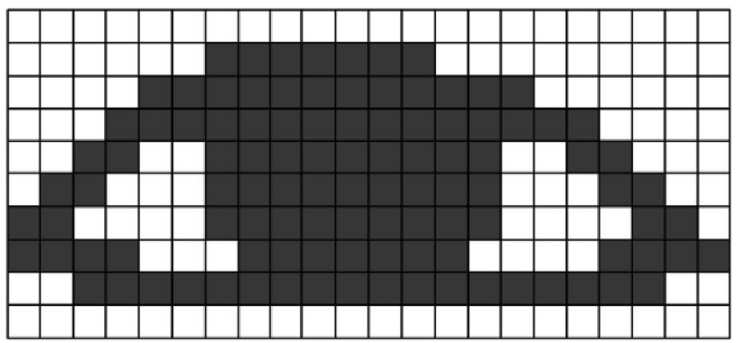

(a)

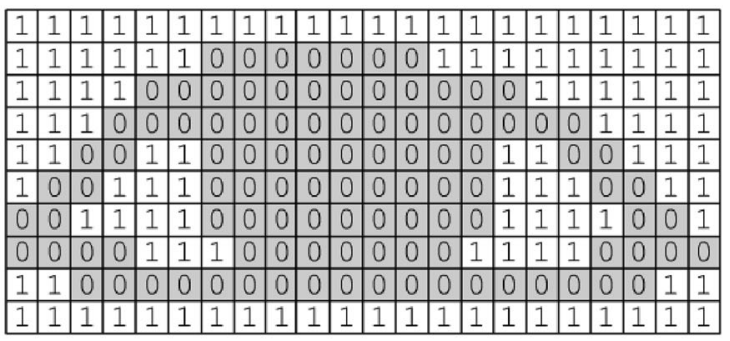

(b)

\begin{tabular}{|l|l|l|l|l|l|l|l|l|l|l|}
\hline 1 & 0 & 0 & 0 & 0 & 0 & 0 & 0 & 0 & 0 & 1 \\
\hline 1 & 0 & 0 & 0 & 0 & 0 & 0 & 0 & 0 & 0 & 1 \\
\hline
\end{tabular}

(c)

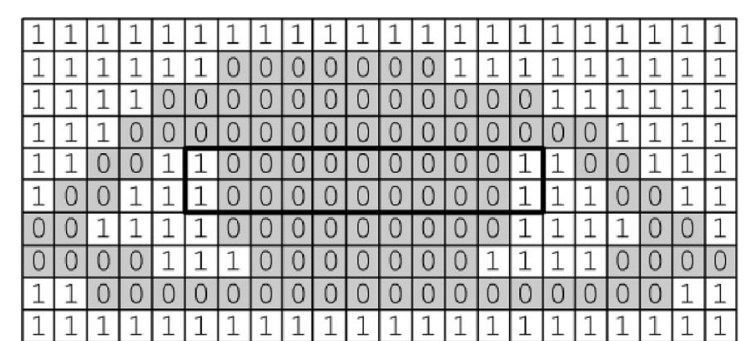

(d)

\begin{tabular}{|c|c|c|c|c|c|c|c|c|c|c|c|c|c|c|c|c|c|c|c|}
\hline & & & & & & & & & & & & & & & & & & & \\
\hline & 0 & 5 & \begin{tabular}{l|l}
1 & 1
\end{tabular} & 1 & 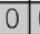 & 0 & & & & & & & & 0 & ] & 0 & & & \\
\hline & & \begin{tabular}{l|l}
00 \\
\end{tabular} & & 0 & & & & & & & & & & 0 & & 0 & & 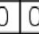 & \\
\hline & & & & 0 & & & & & & & & & & 0 & & & & & \\
\hline & & & & 1 & & 0 & ] & 0 & & & & & 1 & 0 & & 0 & & & \\
\hline 0 & $\overline{0}$ & 00 & & 1 & & & & J & & & & & 1 & 0 & & 0 & & & \\
\hline & $\overline{0}$ & $\overline{0} 0$ & 5 & 1 & $\overline{0}$ & 0 & & & & & & & 1 & 0 & 0 & 0 & & & \\
\hline 0 & 0 & \begin{tabular}{l|l}
0 & 0 \\
\end{tabular} & 5 & 1 & 0 & 0 & & & & & & & 1 & 0 & 0 & 0 & & & \\
\hline & & $\underline{0}$ & 00 & 0 & & & & & & & & & & $\overline{0}$ & & & & & \\
\hline & & & & & & & & & & & & & & & & & & & \\
\hline
\end{tabular}

(e)

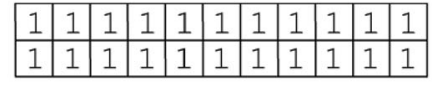

(f)

\begin{tabular}{|c|c|c|c|c|c|c|c|c|c|c|c|c|c|c|c|c|c|}
\hline & & & & & \begin{tabular}{l|l}
1 & 1 \\
\end{tabular} & & & \begin{tabular}{l|l}
1 & 1
\end{tabular} & 11 & 1 & & & & & & & \\
\hline & & 1 & 1 & $\perp$ & \begin{tabular}{|l|l|}
1 & \\
\end{tabular} & \begin{tabular}{|l|l|}
1 & 1 \\
\end{tabular} & 1 & \begin{tabular}{l|l}
1 & 1 \\
\end{tabular} & 1 & 11 & 11 & 1 & & 0 & 0 & & \\
\hline & & 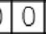 & $\overline{0}$ & 0 & & 00 & 0 & $\begin{array}{lll}0 & 0\end{array}$ & 0 & 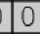 & ju & 0 & & 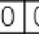 & 0 & 10 & \\
\hline & & 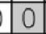 & $\overline{0}$ & $\pi$ & 0 & 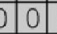 & 8 & & & 0 & 0 & 0 & & & 0 & 0 & 0 \\
\hline & $\overline{0}$ & $\overline{0}$ & $\overline{0}$ & 1 & 7 & 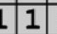 & 1 & $\begin{array}{ll}11 \\
\end{array}$ & 11 &. & 1 & 1 & & & 010 & 0 & 0 \\
\hline C & & 0 & 0 & 1 & 11 & $\begin{array}{lll}1 & 1\end{array}$ & 1 & 1 & $\perp$ & -1 & 14 & 1 & & 0 & 0 & 5 & 0 \\
\hline & \begin{tabular}{l|l}
0 & 0 \\
\end{tabular} & 0 & 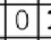 & 1 & \begin{tabular}{l|l}
1 & 1 \\
\end{tabular} & \begin{tabular}{l|l|}
1 & 1 \\
\end{tabular} & $t$ & \begin{tabular}{l|l}
11 \\
\end{tabular} & 11 & 1 & 1 & 1 & & 0 & 0 & & \\
\hline & $\overline{0}$ & 0 & 0 & 1 & \begin{tabular}{|l|l}
1 & 1
\end{tabular} & 11 & 1 & \begin{tabular}{l|l}
11 \\
\end{tabular} & \begin{tabular}{l|l}
1 & 1
\end{tabular} & 1 & 1 & 1 & & 0 & 0 & 0 & \\
\hline & $\overline{0}$ & $\overline{0}$ & $\overline{0}$ & $\overline{0}$ & \begin{tabular}{|l|l|}
0 & 0 \\
\end{tabular} & 00 & + & & & & & & & & & & \\
\hline & & & & & & & & & & & & & & & & & \\
\hline
\end{tabular}

(g)

Figure 8. Iris-sclera pattern analysis: (a) image of eye region, (b) binary representation of the image, (c) proposed structuring element used for opening, (d) opening structuring element placed over eye image, (e) after morphological opening, (f) line structuring element used for closing and (g) localised iris-sclera region obtained after morphological closing.

search in $I_{H R D}$ is reduced to a small region around the most probable face centre determined in the coarse face detection.

Face templates Face templates of direction vectors are used for fine face detection [32]. Templates are created for each size $r$ and for each rotation angle $\phi$. The set of values for $r$ and $\phi$ is denoted by $R$ and $\Phi$, respectively:

$$
R=\left\{r / b_{\min } \leq r \leq b_{\max }\right\},
$$

$$
\Phi=\left\{\phi / \phi_{\min } \leq \phi \leq \phi_{\max }\right\} .
$$

$T_{F R \Phi}$ is an array that stores all the templates for different sizes and angles. The values used for creating $T_{F R \Phi}$ are given by $\left\{b_{\min }, b_{\max }\right\}=\{60$ pixels, 120 pixels $\}$ and $\left\{\phi_{\min }, \phi_{\max }\right\}=\left\{-40^{\circ}, 40^{\circ}\right\}$. Twelve ellipses of different sizes, with a step of five pixels, and rotation steps of $10^{\circ}$ were used. Totally, $12 \times 9$ templates were built for detecting faces. 


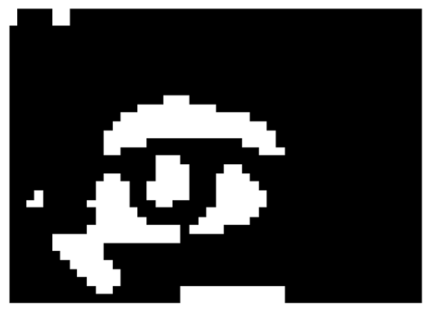

(a)

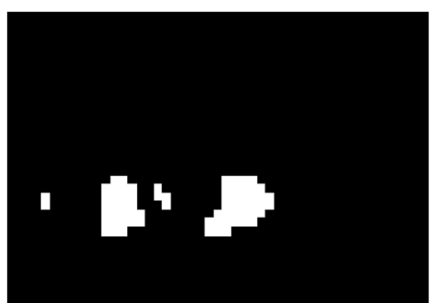

(b)

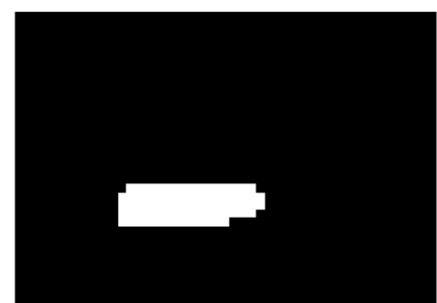

(c)

Figure 9. The open-eye localisation of the preprocessed image (a) after applying threshold, (b) after morphological opening and (c) after morphological closing.

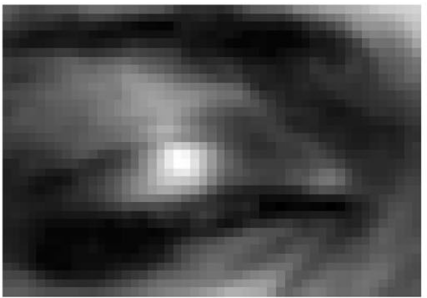

(a)

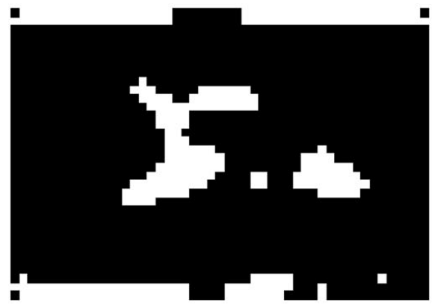

(d)

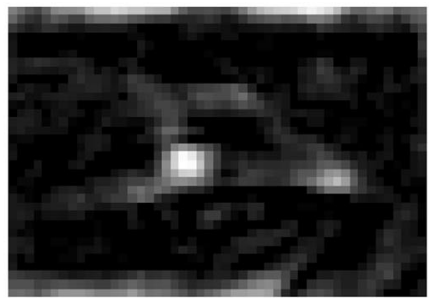

(b)

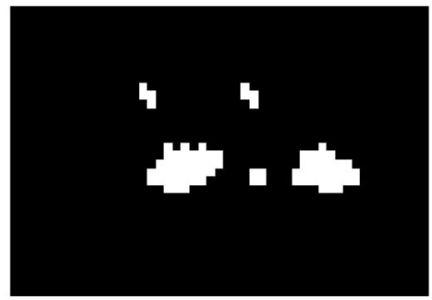

(e)

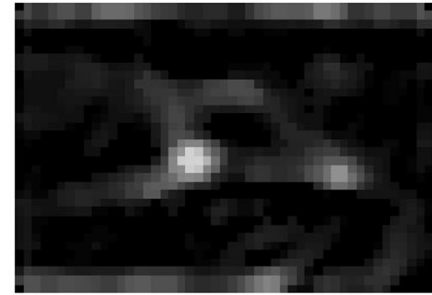

(c)

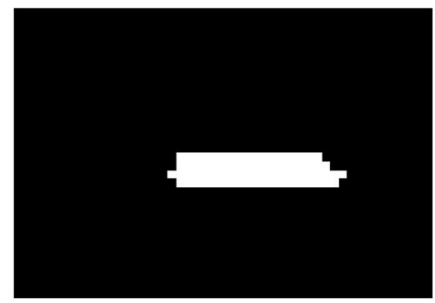

(f)

Figure 10. Iris-sclera detected on a closed eye due to reflections on the eyelid: (a) closed eye region, and after applying (b) tophat, (c) median, (d) threshold, (e) opening and (f) closing.

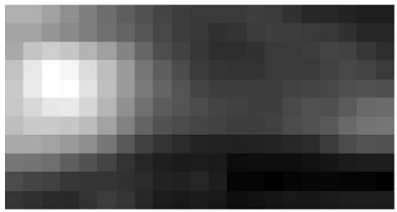

(a)

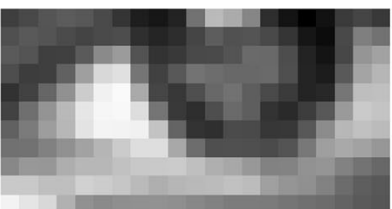

(d)

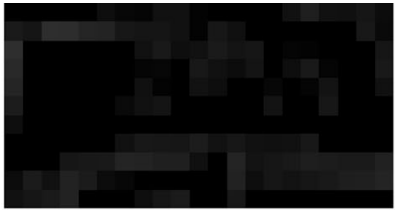

(b)

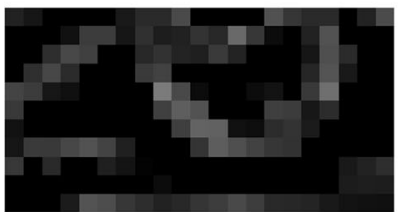

(e)

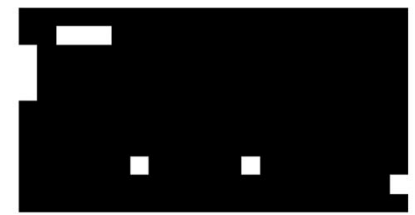

(c)

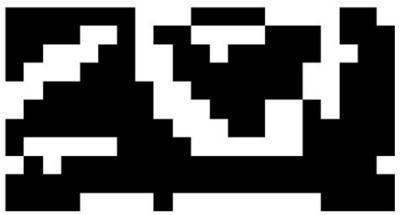

(f)

Figure 11. Confirmation of the presence of open eye $(\mathbf{d}, \mathbf{e}, \mathbf{f})$ by taking laplacian on the iris-sclera detected region: (a, d) iris-sclera region, (b, e) laplacian image and (c, f) edgemap showing the dominant edges on the iris-sclera boundary.

A modified set of templates as shown in figure 3 was used in this system. The modification was driven by the observation that in most of the captured images, the edge of the chin was not clearly differentiated due to the positioning of camera and illumination variation. Thus, the lower half of the elliptical face contour 


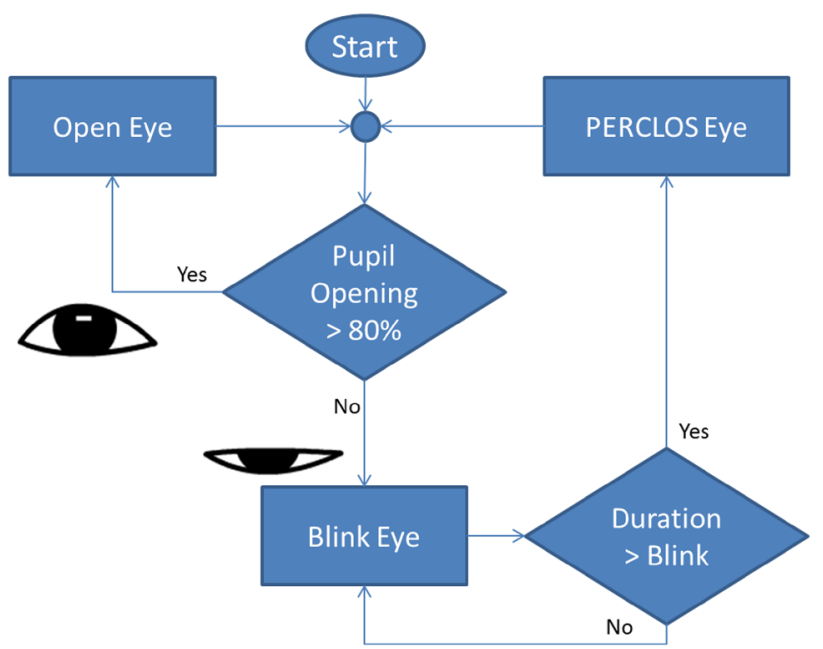

Figure 12. Eye states for PERCLOS estimation.

considered by Maio and Maltoni [32] was removed for this system.

Distinctive anatomical elements in the face region used for creating the templates are eyebrows, nose and lips.
Vectors in the directional image associated with these three anatomical elements follow similar patterns across the population. The angles of direction vectors in the face template for the eyebrows and mouth tend to be horizontal $\left(0^{\circ}\right)$ and for the nose, vertical $\left(90^{\circ}\right)$. These angles are measured relative to a frontal face with no coronal rotation. For faces with coronal rotation by angle $\phi$, the angles in the template are $\phi$ for the eyebrows and mouth, and $90^{\circ}+\phi$ for the nose.

Figure 3 shows the two layers of the templates, $T_{F R \Phi}$, for rotation angles $10^{\circ}, 0^{\circ}$ and $-10^{\circ}$, which are used in computing $I_{T_{F R \Phi}}$. Figure $3 \mathrm{a}-\mathrm{c}$ shows layer1, which is the mask region of templates. The black regions in these figures are ones and white regions surrounding these black regions have a zero. The corresponding direction vectors at each grid position corresponding to the mask regions are stored in layer2, as shown in figure $3 \mathrm{~d}-\mathrm{f}$. These $T_{F R \Phi}$ include the anatomical elements identified with rectangular marks representing the eyebrows, nose and mouth.

Fine detection The $T_{F R \Phi}$ is used to measure its similarity with $I_{R O I}$. A discrete and normalised line integral $I_{T_{F R \Phi}}[32]$ is performed on $I_{R O I}$ for each possible value of the vertical

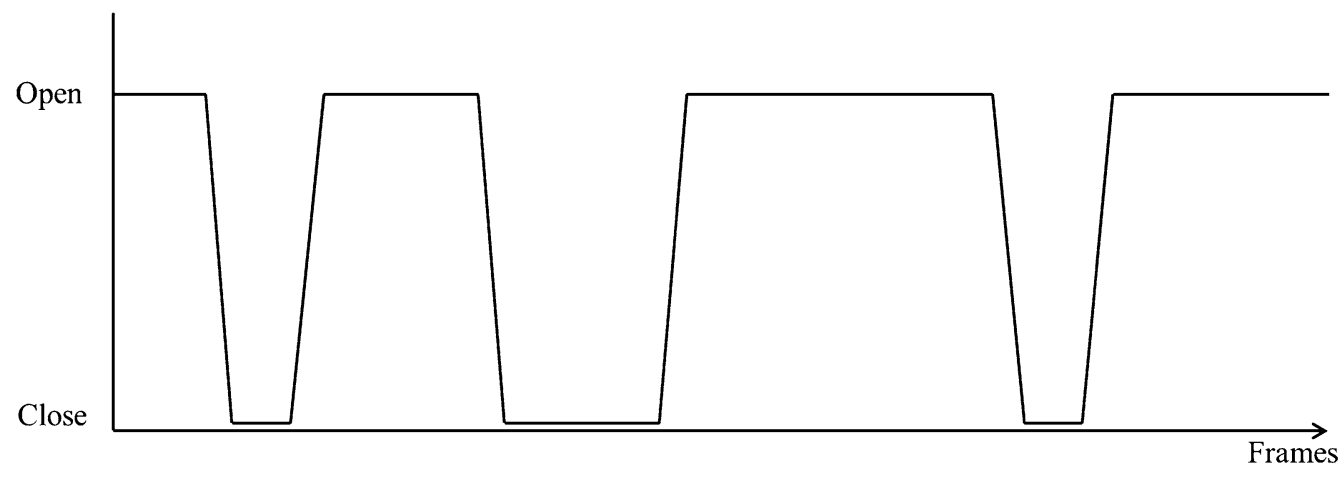

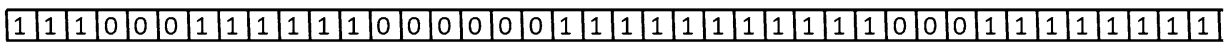

(a)

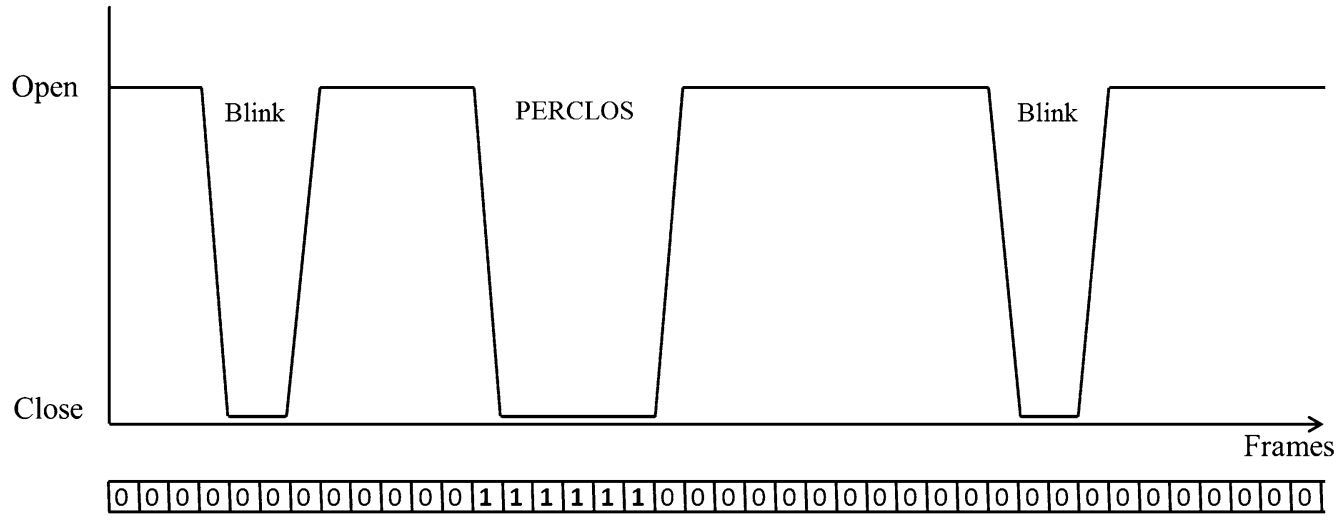

(b)

Figure 13. PERCLOS value computation: (a) frames marked open (1) or closed (0) are shown in the bit stream and (b) PERCLOS computation based on eye-closed duration is shown in the bit stream. 
semi-axis in the set formed by $\left[b_{\min }, \ldots, b_{\max }\right] \times\left[\phi_{\min }, \ldots, \phi_{\max }\right]$. The template position centre that maximises $I_{T_{F R \Phi}}$ is taken as the position for the face centre. The final face region is denoted by $I_{\text {Face }}$. Figure 4 shows the detailed direction vectors and the detected fine face region.

3.1c Eye localisation: The $I_{F a c e}$ region contains only a part of the entire face, which includes the eyebrows, nose and mouth. The size of $I_{\text {Face }}$ was standardised to $128 \times 128$ by resizing using bilinear interpolation. Then, $30 \times 45$ rectangular regions are extracted below each eyebrow at coordinates $(29,25)$ and $(99,25)$ for searching open eye as shown in figure 5 . These values were finalised after analysing over $900 I_{\text {Face }}$ images. An eye region is denoted by I eye_region.

\subsection{Open-eye detection}

The proposed system uses low-resolution images $(640 \times 480)$ and can detect iris as small as 8-12 pixels in diameter.

A new method for detecting an open eye is proposed based on the observation that the sclera appears to be symmetric about the iris as shown in figure 6. By localising and analysing the iris-sclera region, an open eye can be successfully detected from low-resolution images where it is impossible to find the pupil within the iris. As shown in the figure, since the pupil is located at the centre of the iris, approximately $60 \%$ iris is covered by the upper eyelid, which implies that approximately $80 \%$ of the pupil is occluded. Also, as shown, the lower eyelid covers about $10 \%$ of the iris. Thus it can be concluded that $30 \%$ of iris visibility implies approximately $20 \%$ of the pupil is visible. This information is used for drowsiness detection in the proposed system.

The open-eye detection stage consists of three steps: preprocessing, open-eye region localisation and open-eye confirmation.

3.2a Preprocessing: The image $I_{\text {eye_region }}$ may contain the iris, sclera, eyelids and some parts surrounding the eye as shown in figure 5 . The preprocessing on $I_{\text {eye_region }}$ enhances the iris-sclera region (figure 7). The first operation performed on the eye region is a tophat transformation [40] with a disc structuring element $S_{\text {disc }}$ of radius 3 pixels. This leaves behind the bright regions that could be sclera, suppressing all the slowly varying regions, resulting in $I_{\text {tophat }}$. The tophat transform on $I_{\text {eye_region }}$ is the difference between the opened image ( $\circ$ ) and the original image, represented as follows:

$$
\begin{aligned}
I_{\text {tophat }} & =I_{\text {eye_region }}-\left(I_{\text {eye_region }} \circ S_{\text {disc }}\right) \\
& =I_{\text {eye_region }}-\left(I_{\text {eye_region }} \ominus S_{\text {disc }}\right) \oplus S_{\text {disc }}
\end{aligned}
$$

where, for the grey-scale image $I_{\text {eye_region }}$ and the binary structuring element $S_{d i s c}$, erosion operation $(\ominus)$ is given by

$$
\begin{aligned}
I_{\text {eye_region }} \ominus S_{\text {disc }} & =\left[I_{\text {eye_region }} \ominus S_{\text {disc }}\right](x, y) \\
& =\min _{(s, t) \in S_{\text {disc }}}\left\{I_{\text {eye_region }}(x+s, y+t)\right\}
\end{aligned}
$$

and dilation operation $(\oplus)$ is given by

$$
\begin{aligned}
I_{\text {eye_region }} \oplus S_{\text {disc }} & =\left[I_{\text {eye_region }} \oplus S_{\text {disc }}\right](x, y) \\
& =\max _{(s, t) \in S_{\text {disc }}}\left\{I_{\text {eye_region }}(x-s, y-t)\right\} .
\end{aligned}
$$

Some small bright or dark regions may be present in $I_{\text {tophat }}$, which have to be eliminated before applying threshold for better results. This is achieved by applying a median filter on $I_{\text {tophat }}$ using a neighbourhood $\Omega$ of size $k \times k$ as follows:

$$
I_{\text {median }}(x, y)=\operatorname{med}_{(s, t) \in \Omega}\left\{I_{\text {tophat }}(x+s, y+t)\right\}
$$

where $s$ and $t$ vary from $-\left(\frac{k-1}{2}\right)$ to $+\left(\frac{k-1}{2}\right)$.

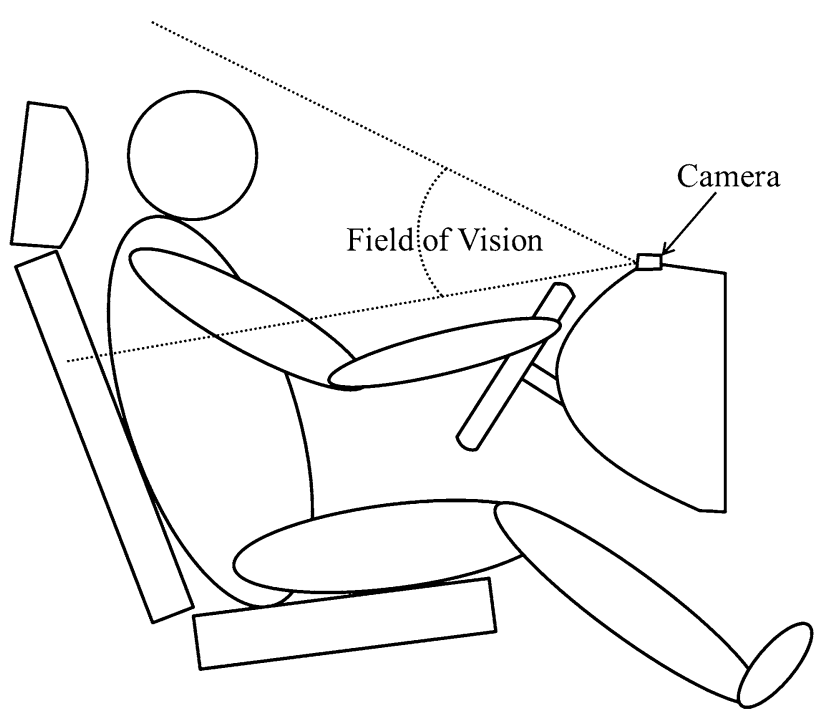

Figure 14. Experimental set-up: placement of camera on the dash board for capturing the image of the driver.

Table 1. Comparison of performance of VJM, GFT, PLE and M-PLE methods.

\begin{tabular}{lcccc}
\hline & $\begin{array}{c}\text { VJM } \\
(\%)\end{array}$ & $\begin{array}{c}\text { GFT } \\
(\%)\end{array}$ & $\begin{array}{c}\text { PLE } \\
(\%)\end{array}$ & $\begin{array}{c}\text { M-PLE } \\
(\%)\end{array}$ \\
\hline $\begin{array}{l}\text { Varying } \\
\quad \text { illumination }\end{array}$ & 93 & 97 & 97 & 98 \\
$\begin{array}{l}\text { Complex } \\
\quad \text { background }\end{array}$ & 95 & 97 & 96 & 98 \\
In lab & 99 & 99 & 99 & 99 \\
Inside car & 93 & 97 & 96 & 98 \\
\hline
\end{tabular}




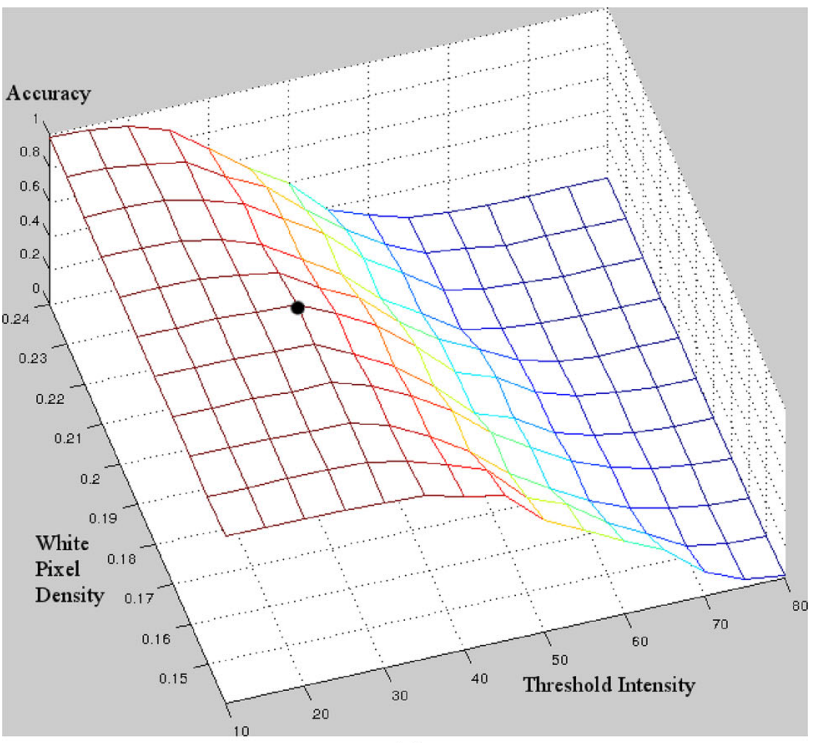

(a)

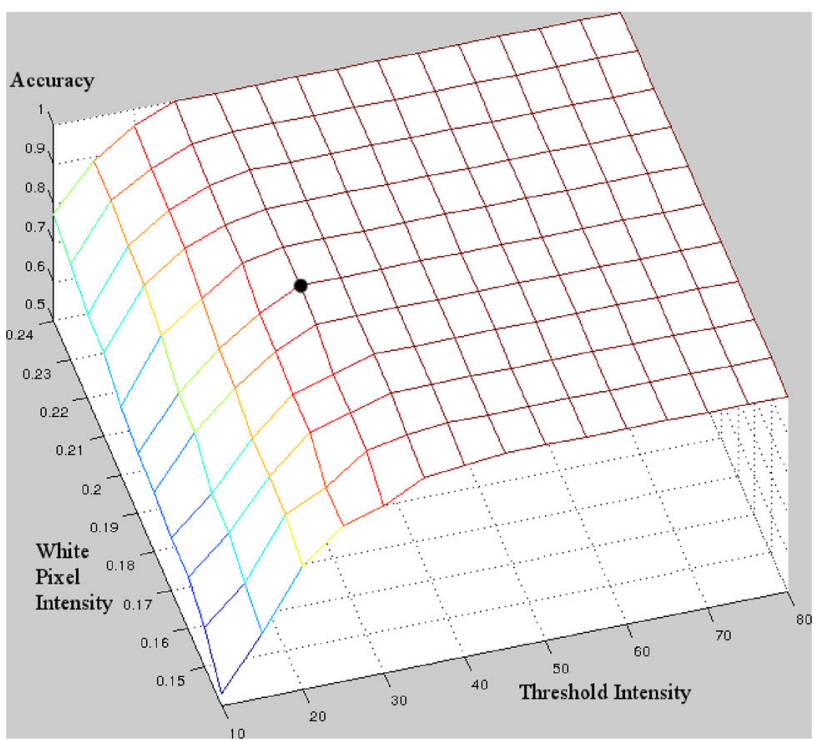

(b)

Figure 15. Selection of $T_{\text {eye }}$ and $T_{\text {white }}$ : threshold intensity $T_{\text {eye }}$ is taken along $x$-axis, white pixel density $T_{\text {white }}$ along $y$-axis and the accuracy for each combination of $T_{\text {eye }}$ and $T_{\text {white }}$ along the $z$-axis. Threshold selection for classifying (a) open eyes and (b) closed eyes.

3.2b Open-eye localisation: The resulting image after the preprocessing will retain only the possible sclera regions. Open eye is detected by searching the symmetry of sclera. For better detection rate of iris-sclera pattern, a threshold is applied on $I_{\text {median }}$ to get $I_{\text {threshold }}$, the thresholded image. The Otsu algorithm [41] is used for determining a good threshold value, $T$.

$$
I_{\text {threshold }}(x, y)=\left\{\begin{array}{cc}
1 & I_{\text {median }}(x, y) \geq T \\
0 & \text { otherwise }
\end{array}\right.
$$

The proposed method for detecting the iris-sclera region consists of a morphological opening operation on the $I_{\text {threshold }}$ using a symmetric structuring element $S_{\text {sclera }}$ (figure 8c) followed by a morphological closing operation using a line structuring element $S_{\text {line }}$ (figure 8f), represented as follows:

$$
I_{\text {sclera_regions }}=\left(I_{\text {threshold }} \circ S_{\text {sclera }}\right) \bullet S_{\text {line }} .
$$

The structure of $S_{\text {sclera }}$ is selected based on the symmetry of the sclera about the iris. The ones in $S_{\text {sclera }}$ (figure 8c) represent the sclera region and zeros represents the iris (a few sclera pixels may fall in this region). Considering the diameter of iris to be 9 pixels long, if 2.7 pixels (approximately 30\%) of sclera region is visible then it can be concluded that eye is open. While designing the structuring element for open-eye localisation (figure 8), taking into consideration the error in applying threshold at the boundaries between eyelids and sclera, the height of structuring element is taken as 2 pixels. The height as well as the length of the structuring element shown in figure 8c should be increased proportionally as the diameter of the iris (in pixels) increases.

Figure 8 gives a detailed explanation of the open-eye localisation process. The resulting image $I_{\text {sclera_regions }}$ will contain the possible eye (iris-sclera) region(s) as shown in figure 9 .

3.2c Open-eye confirmation: As shown in figure 10, reflections on the eyelids can result in adding false sclera

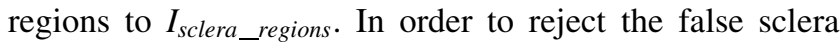
regions that were short-listed as open eye, the iris-sclera vertical boundary is searched using a weighted laplacian operator on the short-listed region(s). The weights of the laplacian are selected such that vertical edges are given more importance. Each $10 \times 20$ region $I_{\text {iris_sclera }}$ in the shortlisted iris-sclera region(s) is taken from $I_{\text {sclera_regions }}$ and the laplacian is applied. As shown in figure 11 , the iris-sclera boundary will be visible in the laplacian image.

$$
\begin{aligned}
I_{\text {eye }}(x, y)= & I_{\text {iris_sclera }}(x-1, y-1) \\
& +I_{\text {iris_sclera }}(x+1, y-1) \\
& +2 I_{\text {iris_sclera }}(x-1, y) \\
& +2 I_{\text {iris_sclera }}(x+1, y) \\
& +I_{\text {iris_sclera }}(x-1, y+1) \\
& +I_{\text {iris_sclera }}(x+1, y+1) \\
& -8 I_{\text {iris_sclera }}(x, y) .
\end{aligned}
$$

This image $I_{\text {eye }}$ is thresholded with a manually set intensity level $T_{\text {eye }}$ to create a binary image $I_{\text {binary_eye }}$. Next, the percentage of white pixels present in the $I_{\text {binary_eye }}$ is considered for confirming whether an eye is open. Since the I binary_eye contains more of vertical edge information, this 
white pixel density represented as $W_{\text {eye }}$ is a good value for open-eye classification. If $W_{\text {eye }}$ is less than a threshold $T_{\text {white}}$, then the eye is classified as closed and otherwise as open.

$$
I_{\text {binary_eye }}(x, y)=\left\{\begin{array}{cc}
1 & I_{\text {eye }}(x, y) \geq T_{\text {eye }} \\
0 & \text { otherwise }
\end{array}\right.
$$

$$
W_{\text {eye }}=\frac{\sum_{(x, y) \in I_{\text {binary_eye }}} I_{\text {binary_eye }}(x, y)}{N_{\text {binary_eye }}}
$$

where $N_{\text {binary_eye }}$ is the number of pixels in $I_{\text {binary_eye }}$.

$$
\text { EyeState }=\left\{\begin{array}{cc}
\text { open } & W_{\text {eye }} \geq T_{\text {white }} \\
\text { closed } & \text { otherwise }
\end{array}\right.
$$

Table 2. Performance of the proposed open-eye detection method on image data set.

\begin{tabular}{lcccrr}
\hline & & Images & Detected open & Detected closed & Accuracy $(\%)$ \\
\hline Varying illumination & Open & 341 & 310 & 31 & 91 \\
& Closed & 192 & 2 & 190 & 99 \\
Complex background & Open & 237 & 226 & 11 & 95 \\
& Closed & 169 & 1 & 168 & 99 \\
\hline
\end{tabular}

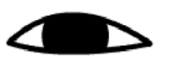

(a)

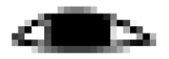

(b)

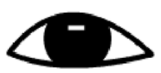

(i)

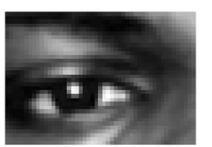

(c)

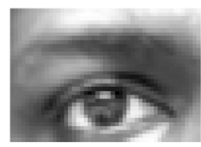

$(\mathbf{k})$

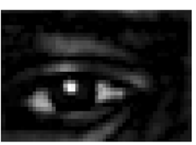

(d)

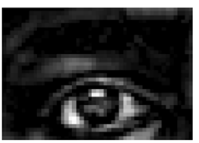

(l)

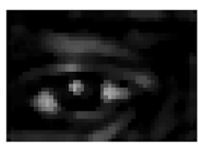

(e)

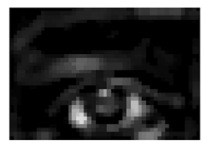

(m)

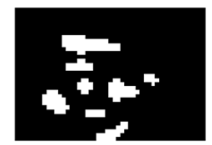

(f)

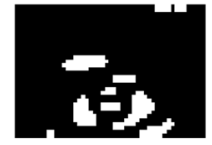

(n)

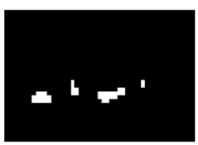

(g)

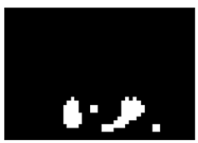

(o)

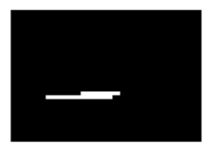

(h)

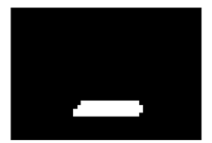

(p)

Figure 16. Normal eye posture while driving on a straight road or a highway. (a, i) The expected postures and (b, j) the expected postures in a low-resolution digital image. (c, k) The original image captured from real life. The processing steps shown are as follows: $(\mathbf{d}, \mathbf{l})$ tophat transformation, (e, m) median filtering, (f, n) thresholding, $(\mathbf{g}, \mathbf{o})$ morphological opening and (h, p) morphological closing.

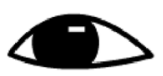

(a)

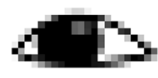

(b)

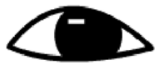

(i)

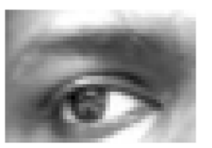

(c)

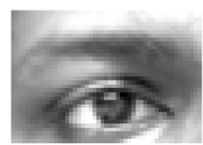

(k)

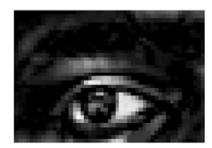

(d)

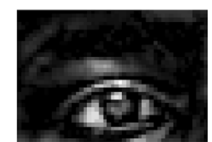

(I)

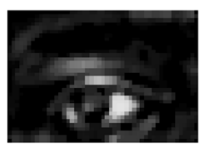

(e)

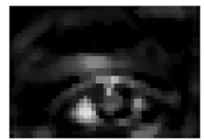

(m)

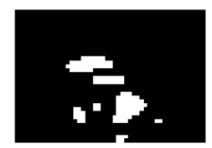

(f)

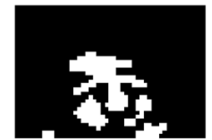

(n)

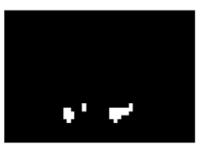

(g)

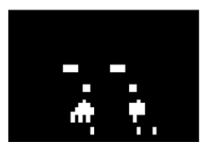

(o)

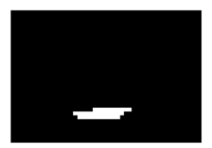

(h)

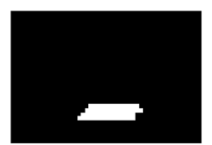

(p)

Figure 17. Eye postures on roads with curves and turns. (a, i) The expected postures and (b, j) the expected postures in a low-resolution digital image. (c, k) The original image captured from real life. The processing steps shown are as follows: (d, l) tophat transformation, $(\mathbf{e}, \mathbf{m})$ median filtering, (f, n) thresholding, (g, o) morphological opening and (h, p) morphological closing. 


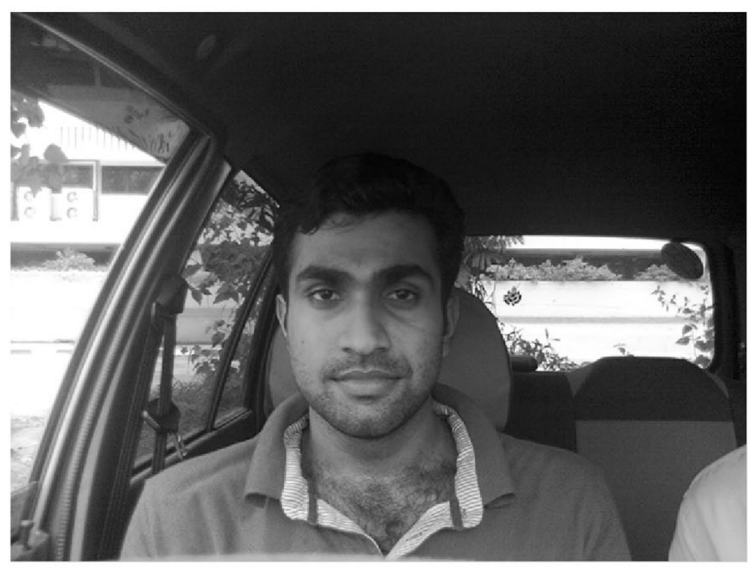

(a)

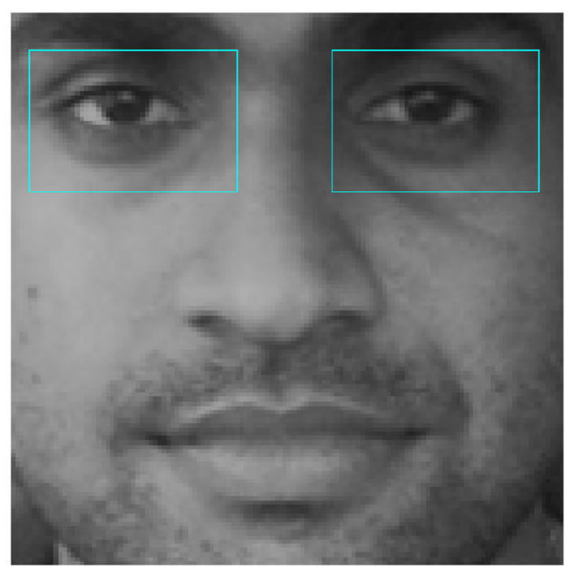

(b)

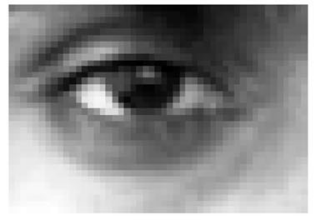

(c)

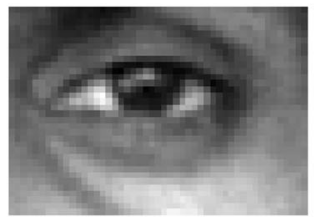

(g)

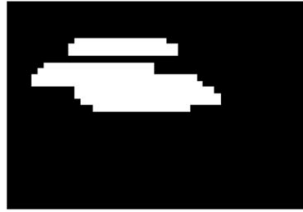

(d)

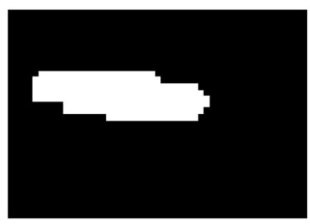

(h)

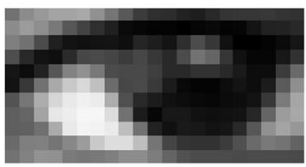

(e)

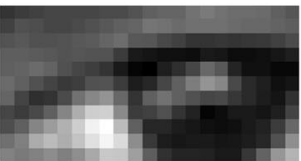

(i)

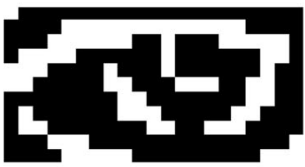

(f)

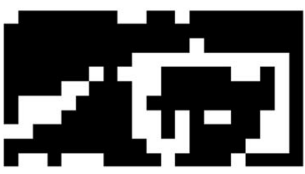

(j)

Figure 18. Open-eye detection: (a) original frame, $(\mathbf{b})$ fine face region, $(\mathbf{c}, \mathbf{g})$ localised eye regions, $(\mathbf{d}, \mathbf{h})$ iris-sclera localisation using morphological closing, (e, i) iris-sclera regions and (f, j) edgemap confirms open eyes.

\subsection{Real-time video processing}

When performing the open-eye detection on a continuous sequence of images, i.e., a video, the coarse face detection is performed on the first frame. Next frame onwards, the coarse face location is assumed to be at the fine face location of the previous frame. This approach is supported by the observation that the change in position of face in two consecutive frames is small.

\subsection{Drowsiness detection}

In the proposed approach, the PERCLOS system is used for determining the driver drowsiness. The PERCLOS algorithm calculates the proportion of time that the eyelid covers $80 \%$ of the pupil [42]. The proposed open-eye detection method includes this part of the PERCLOS, i.e., the open-eye detection will detect only those eyes in which at least $30 \%$ of iris is visible as explained in section 3.2.

When measuring the drowsiness, classifying the eye blink and the PERCLOS is an important step for a better prediction. Hence, in this system, up to four contiguous frames of closed eye are considered as a blink state and any sequence of more than 4 frames with closed eye is considered as a PERCLOS state, as shown in figure 12. Based on the eye state and duration of closed eye, the upcoming sleep is predicted, i.e., if more than $50 \%$ of the time the system is in PERCLOS state, the driver is drowsy. In this implementation the PERCLOS pattern is analysed every 30 s. Eye state is determined for each frame, and the frames identified to be in PERCLOS state are marked with 'one' and other frames are marked 'zero' as shown in figure 13. 


\section{Experimental results}

In the experimental set-up, the camera for capturing the driver was placed on the dash board just above the steering wheel as shown in figure 14 . A web-cam with $640 \times 480$ pixels resolution and a speed of 15 frames per second were used. The processing hardware used is a Pentium 4, 1.8 GHz machine with 1 GB RAM. The system was modelled using MATLAB 7.

\subsection{Test data}

The initial experiments were conducted on standard face databases. However, these images were taken in a studio environment and lacked the illumination variations and complex backgrounds. Hence, a data set was created by capturing real-life images using the set-up shown in figure 14.

The newly created data set consists of 939 images and 23 videos of 12 different subjects. These images and videos cover different skin-tones, illuminations, heights of the driver and changing backgrounds taken inside the vehicle and in the lab. The images inside the vehicle were taken at different times of day from morning to evening across different weather conditions and different vehicles.

\subsection{Results}

The tests were performed in two steps: face detection and open-eye detection. The face detection was compared using VJM, GFT [29], PLE, and PLE with modified templates (M-PLE) methods. Major factors considered in selecting the method that best suits the driver drowsiness detection system were the accuracy in detection and computational complexity. The illumination varies greatly inside a vehicle, which makes selecting a good training data set for VJM a challenging task. As shown in table 1, it was observed that GFT and M-PLE methods perform the best in varying

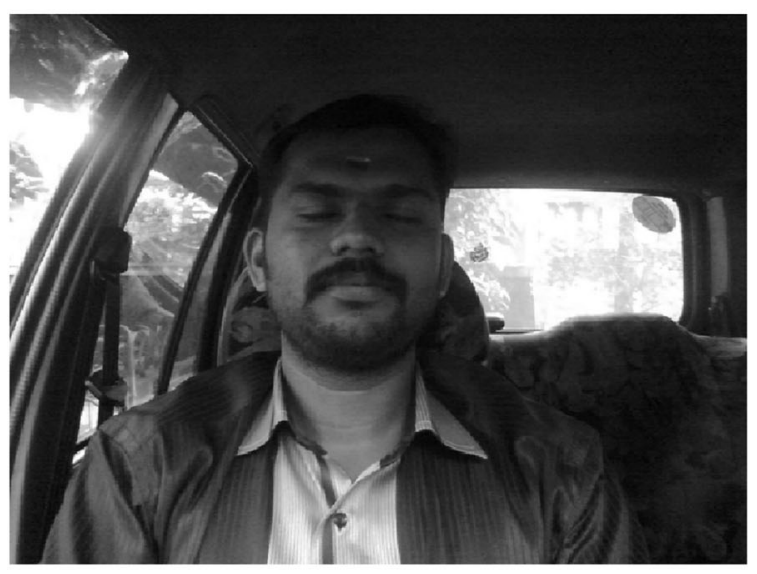

(a)

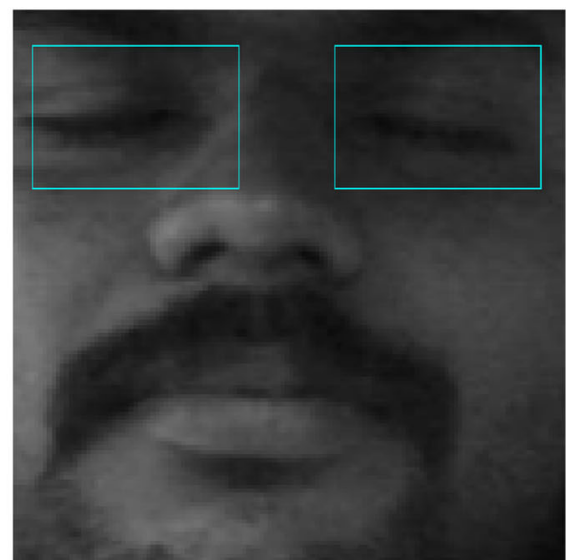

(b)

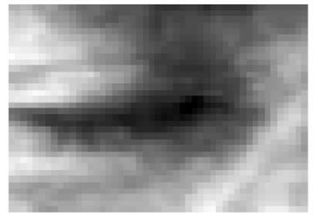

(c)

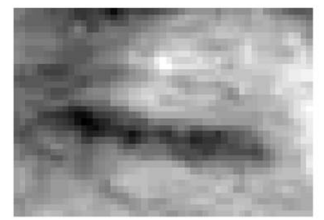

(g)

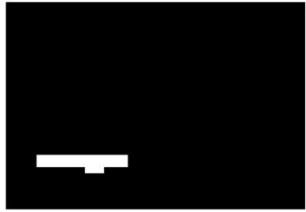

(d)

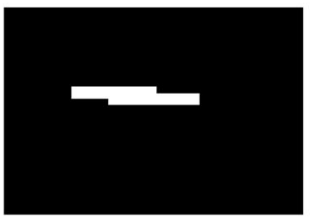

(h)

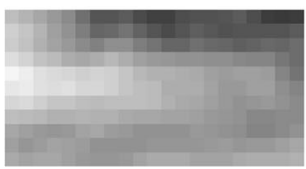

(e)

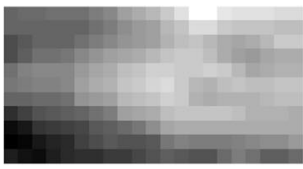

(i)

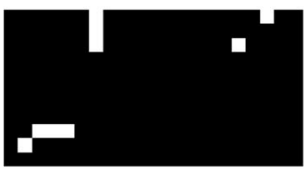

(f)

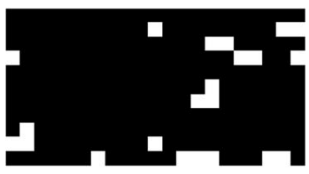

(j)

Figure 19. Closed eyes detection: (a) original frame, (b) fine face region, $(\mathbf{c}, \mathbf{g})$ localised eye regions, (d, h) iris-sclera localisation using morphological closing, $(\mathbf{e}, \mathbf{i})$ iris-sclera regions and $(\mathbf{f}, \mathbf{j})$ edgemap confirms closed eyes. 
illumination conditions and complex background. Considering the fact that the number of computations required for GFT (Gabor filters of at least four orientations and scales have to be used) is much higher than that for M-PLE (direction vectors are computed for coarse and fine face detection), M-PLE was chosen for face detection.

The threshold values $T_{\text {eye }}$ and $T_{\text {white }}$ in the open-eye confirmation step were carefully selected after performing tests on more than 680 images of open and closed eyes. The findings are summarised in figure 15 . On the resulting $I_{\text {binary_eye }}$, the percentage of white pixels, $W_{\text {eye }}$, is computed. It was found that the open and closed eyes are satisfactorily classified when the $T_{\text {eye }}$ is 30 (on 255 grey levels) and the $T_{\text {white }}$ is $0.19(19 \%)$. These points are marked in figure 15. It was found that at these values the accuracy of closed eye detection is $100 \%$ and open eye is $97 \%$. Since the priority is for detecting closed eyes, these values for $T_{\text {eye }}$ and $T_{\text {white }}$ were chosen.
As shown in table 2, a few open-eye images were detected as closed by this method. When these images were analysed, the sclera symmetry about the iris could not be found since the iris was towards the right or left of the eye. Also, the closed eyes detected as open were observed to be severely affected by the reflections on a bright sunny day. As expected, the closed eye detection accuracy is almost 99\%. Even though noises could affect the open-eye detection, still the proposed method achieves an accuracy of $93 \%$ on average.

The various possible cases of iris-sclera pattern are shown in figures 16 and 17. Figure 16 shows the normal eye posture when driving on a straight road. Figure 17 shows the normal postures on roads with curves. The entire process for detecting the iris-sclera region is shown step by step in these figures.

Figure 18 shows the major steps for open-eye detection. Experiments were carried out with varying illumination,

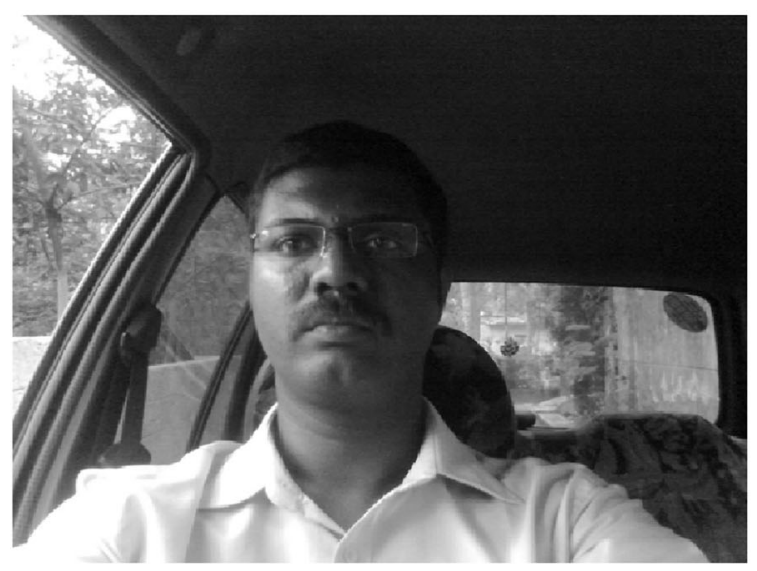

(a)

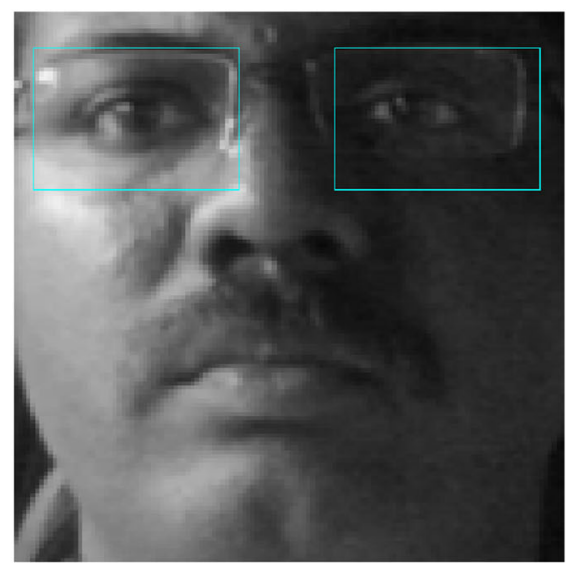

(b)

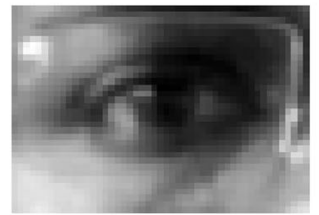

(c)

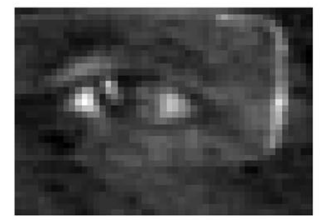

(g)

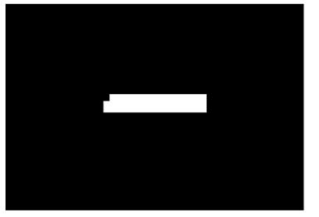

(d)

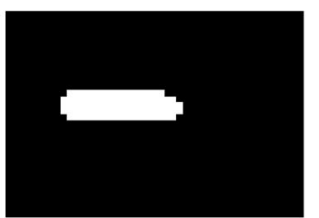

(h)

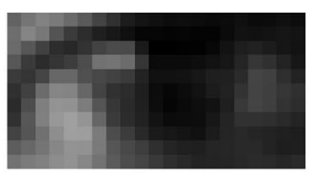

(e)

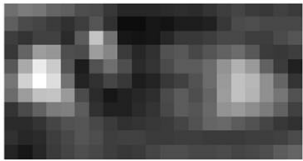

(i)

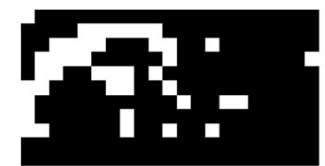

(f)

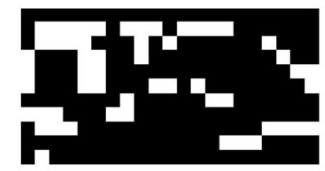

(j)

Figure 20. Presence of spectacles: (a) original frame, (b) fine face region, $(\mathbf{c}, \mathbf{g})$ localised eye regions, (d, h) iris-sclera localisation using morphological closing, (e, i) iris-sclera regions and (f, $\mathbf{j})$ edgemap confirms open eyes. 
Table 3. Performance of the proposed open-eye detection method on video data set.

\begin{tabular}{|c|c|c|c|c|c|}
\hline & & Frames & Detected open & Detected closed & Accuracy $(\%)$ \\
\hline \multirow[t]{2}{*}{ Driver 1} & Open & 611 & 593 & 18 & 97 \\
\hline & Closed & 89 & 0 & 89 & 100 \\
\hline \multirow[t]{2}{*}{ Driver 2} & Open & 421 & 402 & 19 & 95 \\
\hline & Closed & 147 & 4 & 143 & 98 \\
\hline \multirow[t]{2}{*}{ Driver 3} & Open & 304 & 296 & 8 & 97 \\
\hline & Closed & 105 & 0 & 105 & 100 \\
\hline \multirow[t]{2}{*}{ Driver 4} & Open & 529 & 507 & 22 & 96 \\
\hline & Closed & 257 & 2 & 255 & 99 \\
\hline \multirow[t]{2}{*}{ Driver 5} & Open & 384 & 369 & 15 & 96 \\
\hline & Closed & 89 & 3 & 86 & 96 \\
\hline \multirow[t]{2}{*}{ Driver 6} & Open & 931 & 886 & 45 & 95 \\
\hline & Closed & 177 & 5 & 172 & 97 \\
\hline \multirow[t]{2}{*}{ Driver 7} & Open & 391 & 385 & 6 & 98 \\
\hline & Closed & 87 & 0 & 87 & 100 \\
\hline \multirow[t]{2}{*}{ Driver 8} & Open & 213 & 207 & 6 & 97 \\
\hline & Closed & 43 & 0 & 43 & 100 \\
\hline \multirow[t]{2}{*}{ Driver 9} & Open & 473 & 454 & 19 & 96 \\
\hline & Closed & 311 & 5 & 306 & 98 \\
\hline \multirow[t]{2}{*}{ Driver 10} & Open & 856 & 821 & 35 & 96 \\
\hline & Closed & 212 & 1 & 211 & 99 \\
\hline \multirow[t]{2}{*}{ Driver 11} & Open & 657 & 638 & 19 & 97 \\
\hline & Closed & 123 & 2 & 120 & 98 \\
\hline \multirow[t]{2}{*}{ Driver 12} & Open & 453 & 432 & 21 & 95 \\
\hline & Closed & 95 & 0 & 95 & 100 \\
\hline \multirow[t]{2}{*}{ Total } & Open & 6223 & 6011 & 212 & 96 \\
\hline & Closed & 1735 & 22 & 1713 & 99 \\
\hline
\end{tabular}

Table 4. Performance evaluation of the proposed system on video data set.

\begin{tabular}{lccc}
\hline & $\begin{array}{c}\text { Face detection } \\
(\%)\end{array}$ & $\begin{array}{c}\text { Open-eye detection } \\
(\%)\end{array}$ & $\begin{array}{c}\text { Total } \\
(\%)\end{array}$ \\
\hline Driver 1 & 99 & 97 & 96 \\
Driver 2 & 99 & 95 & 94 \\
Driver 3 & 95 & 97 & 92 \\
Driver 4 & 99 & 96 & 95 \\
Driver 5 & 99 & 96 & 95 \\
Driver 6 & 100 & 95 & 95 \\
Driver 7 & 97 & 98 & 95 \\
Driver 8 & 99 & 97 & 96 \\
Driver 9 & 96 & 96 & 92 \\
Driver 10 & 98 & 96 & 94 \\
Driver 11 & 99 & 97 & 96 \\
Driver 12 & 99 & 95 & 94 \\
\hline
\end{tabular}

complex background, in car and in lab on different persons, as shown in figures 18, 19, 20 and 21. As shown in figure 20, the proposed method can detect open eyes even when a person is wearing normal glasses. Since the proposed system uses a visible spectrum camera, this system fails to detect eyes of a person wearing sunglasses. The performance under varying illumination can also be understood clearly form figure 20 .

As shown in table 3, the proposed method was tested on 12 different subjects. A few open eyes looking at the rear view mirrors were detected as closed eyes due to the absence of sclera symmetry. Also, a few closed eyes were mis-detected as open eyes due to the long exposure time while the eyelid was opening. The camera could not capture the eye blink completely. From these frames, the eye state cannot be determined.

The performance of the entire system on combining the face detection and open-eye detection is shown in table 4. The proposed system has achieved an overall accuracy of more than $95 \%$ in 7 of the 12 subject videos. The overall accuracy was computed to be the product of face detection accuracy and open-eye detection accuracy on the frames where face detection was successful.

\subsection{Observations}

One important observation is that, when the driver looks at the outside rear view mirror, the iris will be to the extreme left or right within the eye as shown in figure 21. In this case, the ISPA method fails to detect the sclera symmetry. Prolonged staring at the mirrors could be treated as the inattention of the driver. 


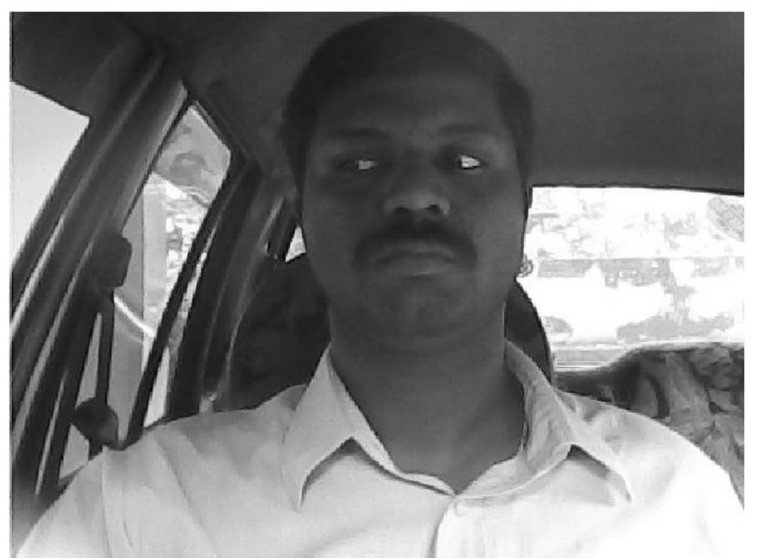

(a)

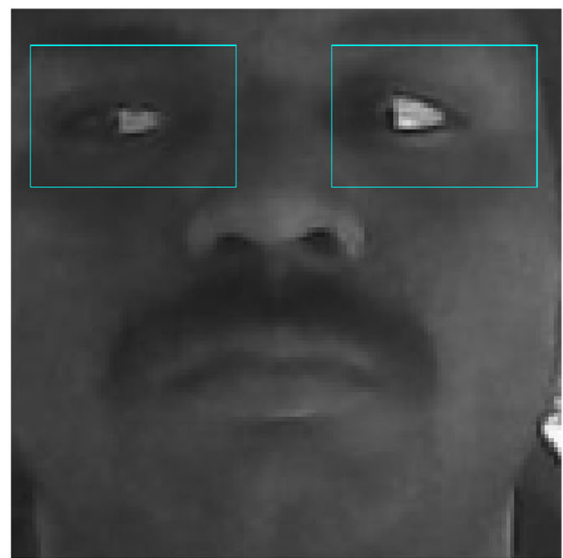

(b)

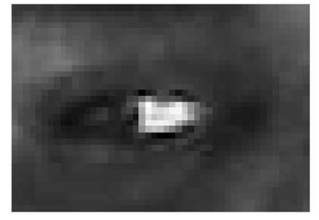

(c)

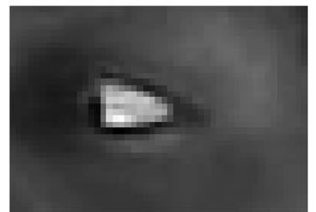

$(\mathbf{g})$

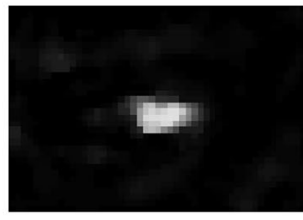

(d)

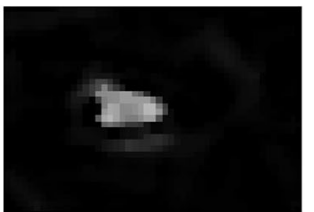

(h)

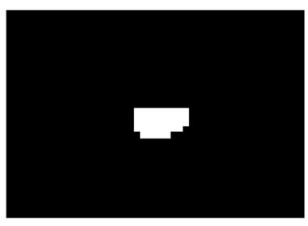

(e)

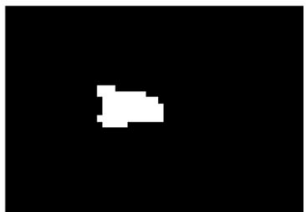

(i)

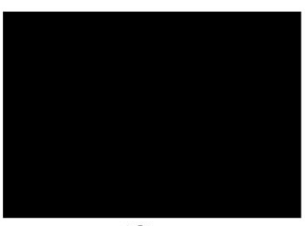

(f)

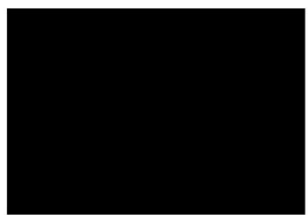

(j)

Figure 21. Mis-detection of open eyes: (a) original frame, (b) fine face region, $(\mathbf{c}, \mathbf{g})$ localised eye regions, $(\mathbf{d}, \mathbf{h})$ median filtering, $(\mathbf{e}$, i) thresholding and $(\mathbf{f}, \mathbf{j})$ iris-sclera regions are missing in the morphological opened image.

\section{Conclusion and future works}

A new method using ISPA is proposed for open-eye detection to be used in vehicle driver drowsiness monitoring. The performance of the proposed ISPA method is satisfactory and it could be extended for night time system using an IR camera with no or a little modification. The ISPA method is proven to work efficiently in low-resolution images for many eye postures. This method yields excellent results on images with varying illumination and complex backgrounds.

The future extension of this work includes the integration of information from vehicle CAN like speed, steering angle, etc. for better prediction of drowsiness.

\section{Acknowledgements}

The authors would like to thank the experts from Tata Elxsi, Technopark, Trivandrum, India, for the support and guidance.

\section{References}

[1] Klauer S G, Dingus T A, Neale V L, Sudweeks J D and Ramsey D J 2006 The impact of driver inattention on nearcrash/crash risk: an analysis using the 100-car naturalistic driving study data. Washington, DC: U.S. Department of Transportation, National Highway Traffic Safety Administration, https://vtechworks.lib.vt.edu/bitstream/handle/10919/ 55090/DriverInattention.pdf?sequence $=1$. Report No. DOT HS 810594

[2] Akerstedt T, Kecklund G and Hörte L G 2001 Night driving, season, and the risk of highway accidents. Sleep 24: 401-406. https://www.ncbi.nlm.nih.gov/pubmed/11403524

[3] Connor J, Norton R, Ameratunga S, Robinson E, Civil I, Dunn R, Bailey J and Jackson R 2002 Driver sleepiness and risk of serious injury to car occupants: population based control study. Br. Med. J. 324: 1125-1129

[4] Horne J and Reyner L 1999 Vehicle accidents related to sleep: a review. Occup. Environ. Med. 56: 189-294

[5] May J F and Baldwin C L 2009 Driver fatigue: the importance of identifying causal factors of fatigue when 
considering detection and countermeasure technologies. Transp. Res. Part F: Traf. Psychol. Behav. 12(3): 218-224

[6] Wright N, Stone B, Horberry T and Reed N 2007 A review of in-vehicle sleepiness detection devices. Published Project Report PPR157, TRL Limited

[7] Bergasa L M, Nuevo J, Sotelo M Á, Barea R and Guillén M E L 2006 Real-time system for monitoring driver vigilance. IEEE Trans. Intell. Transp. Syst. 7(1): 63-77

[8] Oron-Gilad T, Ronen A and Shinar D 2008 Alertness maintaining tasks (AMTs) while driving. Accid. Anal. Prev. 40(3): 851-860

[9] Papadelis C, Chen Z, Kourtidou-Papadeli C, Bamidis P, Chouvarda I, Bekiaris E and Maglaveras N 2007 Monitoring sleepiness with on-board electrophysiological recordings for preventing sleep-deprived traffic accidents. Clin. Neurophysiol. 118(9): 1906-1922

[10] Faber J 2004 Detection of different levels of vigilance by EEG pseudospectra. Neural Netw. World 14(3-4): 285-290

[11] Lin C T, Chang C J, Lin B S, Hung S H, Chao C F and Wang I J 2010 A real-time wireless brain-computer interface system for drowsiness detection. IEEE Trans. Biomed. Circuits Syst. 4(4): 214-222

[12] Wakita T, Ozawa K, Miyajima C, Igarashi K, Itou K, Takeda $\mathrm{K}$ and Itakura F 2006 Driver identification using driving behavior signals. IEICE Trans. E89-D(3): 1188-1194

[13] Takei Y and Furukawa Y 2005 Estimate of drivers fatigue through steering motion. In: Proceedings of the IEEE International Conference on Systems, Man and Cybernetics, vol. 2, pp. 1765-1770

[14] McCall J C, Trivedi M M, Wipf D and Rao B 2005 Lane change intent analysis using robust operators and sparse bayesian learning. In: Proceedings of the 2005 IEEE Computer Society Conference on Computer Vision and Pattern Recognition (CVPR'05). Washington, DC, USA: IEEE Computer Society, p. 59

[15] Chang T H, Hsu C S, Wang C and Yang L K 2008 Onboard measurement and warning module for irregular vehicle behavior. IEEE Trans. Intell. Transp. Syst. 9(3): 501-513

[16] D'Orazio T, Leo M, Guaragnella C and Distante A 2007 A visual approach for driver inattention detection. Pattern Recogn. 40(8): 2341-2355

[17] Suzuki M, Yamamoto N, Yamamoto O, Nakano T and Yamamoto S 2006 Measurement of driver's consciousness by image processing - a method for presuming driver's drowsiness by eye-blinks coping with individual differences. In: Proceedings of the IEEE International Conference on Systems, Man and Cybernetics, vol. 4, pp. 2891-2896

[18] Ji Q and Yang X J 2002 Real-time eye, gaze, and face pose tracking for monitoring driver vigilance. Real-Time Imag. 8(5): $357-377$

[19] Bretzner L and Krantz M 2005 Towards low-cost systems for measuring visual cues of driver fatigue and inattention in automotive applications. In: Proceedings of the IEEE International Conference on Vehicular Electronics and Safety, pp. 161-164

[20] Heinzmann J, Tate D and Scott R 2008 Using technology to eliminate drowsy driving. In: Proceedings of the SPE International Conference on Health, Safety, and Environment in Oil and Gas Exploration and Production

[21] Yang M, Kriegman J and Ahuja N 2002 Detecting faces in images: a survey. IEEE Trans. Pattern Anal. Mach. Intell. 24(1): $34-58$
[22] Dowall J, Pavlidis I and Bebis G 2001 Face detection in the near-IR spectrum. Image Vis. Comput. 21: 565-578

[23] Zhu Z and Ji Q 2005 Robust real-time eye detection and tracking under variable lighting conditions and various face orientations. Comput. Vis. Image Und. 98: 124-154

[24] Perez C A, González G D, Medina L E and Galdames F J 2005 Linear vs. nonlinear neural modeling for 2-D pattern recognition. IEEE Trans. Syst. Man Cybern. Part A: Syst. Hum. 35(6): 955-964

[25] Rowley H, Baluja S and Kanade T 1998 Neural networkbased face detection. IEEE Trans. Pattern Anal. Mach. Intell. 20(1): 23-38

[26] Rowley H, Baluja S and Kanade T 1998 Rotation invariant neural network based face detection. In: Proceedings of the IEEE Computer Society Conference on Computer Vision and Pattern Recognition, Santa Barbara, CA, pp. 38-44

[27] Jones M and Rehg J 2002 Statistical color models with application to skin detection. Int. J. Comput. Vis. 46: 81-96

[28] Wang J G and Sung E 2002 Study on eye gaze estimation. IEEE Trans. Syst. Man Cybern. Part B: Cybern. 32(3): 332-350

[29] Chen Y W and Kubo K 2007 A robust eye detection and tracking technique using Gabor filters. In: Proceedings of the Third International Conference on Intelligent Information Hiding and Multimedia Signal Processing, vol. 1, pp. 109-112

[30] Ji Q 2002 3D face pose estimation and tracking from a monocular camera. Image Vis. Comput. 20(7): 499-511

[31] Li Y, Qi X and Wang Y 2001 Eye detection by using fuzzy template matching and feature-parameter-based judgement. Pattern Recogn. Lett. 22(10): 1111-1124

[32] Maio D and Maltoni D 2000 Real-time face location on grayscale static images. Pattern Recogn. 33: 1525-1539

[33] Viola P and Jones M 2001 Rapid object detection using a boosted cascade of simple features. In: Proceedings of the IEEE Computer Society Conference on Computer Vision and Pattern Recognition, vol. 1, pp. I-511-I-518

[34] Saradadevi M and Bajaj P 2008 Driver fatigue detection using mouth and yawning analysis. Int. J. Comput. Sci. Netw. Secur. 8: 183-188

[35] Perez C A, Lazcano V A and Estévez P A 2007 Real-time iris detection on coronal-axis-rotated faces. IEEE Trans. Syst. Man Cybern. Part C: Appl. Rev. 37(5): 971-978

[36] Liu D, Sun P, Xiao Y and Yin Y 2010 Drowsiness detection based on eyelid movement. In: Proceedings of the Second International Workshop on Education Technology and Computer Science, vol. 2, pp. 49-52

[37] Tabrizi P R and Zoroofi R A 2008 Open/closed eye analysis for drowsiness detection. In: Proceedings of the First Workshops on Image Processing Theory, Tools and Applications, pp. 1-7

[38] Flores M J, Armingol J M and de la Escalera A 2010 Realtime warning system for driver drowsiness detection using visual information. J. Intell. Robot. Syst. 59(2): 103-125

[39] Donahue M J and Rokhlin S I 1993 On the use of level curves in image analysis. In: Image understanding, vol. 57, pp. 185-203

[40] Gonzalez R C and Woods R E 2009 In: Digital image processing. 3rd Ed, Prentice Hall

[41] Otsu N A 1998 Threshold selection method from gray-level histograms. In: IEEE Trans. Syst. Man Cybern. 9(1): 62-66

[42] Dinges D F and Grace R 1998 PERCLOS: a valid psychophysiological measure of alertness as assessed psychometer vigilance. In: US Department of Transportation: Federal Highway Administration 